\section{DIELECTRIC MEASUREIMENTS ON PURE AND KDP ADDED DISODIUIM HYDROGEN PHOSPHATE (DSHP) SINGLE CRYSTALS}

KEY WORDS: Crystal growth Dielectric constant; Dielectric loss, AC Conductivity, DC conductivity, Electrical properties, slow evaporation method.

\section{Dr. J. Asbalter}

Dr. S. Mugundakumari

\section{Dr. N. Joseph} John*
Department of Physics, Kamarajar Government Arts College, Surandai 627859, Tenkasi District, Tamil Nadu, India

Department of Physics, Thiru Kolanjiappar Government Arts College, Cuddalore-626001, Cuddalore District, Tamil Nadu, India

Department of Physics, Kamarajar Government Arts College, Surandai 627859, Tenkasi District,Tamil Nadu, India.*Corresponding Author

Electrical conductivity is an elegant experimental tool to probe the structural defects and internal purity of crystalline solids. In the present study we have grown pure and KDP added DSHP single crystals by the slow evaporation method from aqueous solutions at room temperature. Good quality transparent crystals have been obtained. Melting point and density measurements were done. Electrical conductivity measurements were carried out with two frequencies, $100 \mathrm{~Hz}$ and $1 \mathrm{kHz}$ at various temperatures ranging from 2 to $30 \circ \mathrm{C}$ by using the parallel plate capacitor method. The present study indicate that the dielectric constant and $\mathrm{AC}$ and DC conductivities increase with increase of temperature.

\section{INTRODUCTION}

Electrical conductivity is an elegant experimental tool to probe the structural defects and internal purity of crystalline solids. Most of the earlier investigations on alkali halides rare earth tungstates divalent vanadates phosphates and oxalates and other ferroelectric materials described the electrical conductivity in terms of electrons (or holes), polarons, impurities and thereby the mechanism of ionic conductivity was established on firm footing [1-10].

The electrical conductivity for ionic materials is found to be very low which may be due to the trapping of some carriers at defect sites. At a particular temperature, however, the Gibb's free energy of a crystal is minimum when a certain fraction of ions leaves the normal lattice. As the temperature rises, more and more defects are produced which, in turn, increase the conductivity[10]. In the high temperature (intrinsic) region, the effect of impurity on electrical conduction will not change appreciably whereas in the low temperature (extrinsic) region, the presence of impurity in the crystal increases its conductivity. The electrical conduction in dielectrics is mainly a defect controlled process in the low temperature region. The presence of impurities and vacancies mainly determines this region. The energy needed to form the defect is much larger than the energy needed to form the defect is much larger than the energy needed for its drift $[11,12]$. The conductivity of the crystalline material in the higher temperature region is determined by the intrinsic defects caused by the thermal fluctuations in the crystal.

Disodium hydrogen orthophosphate ( DSHP) is one of the very good NLO materials of phosphate group. It belongs to hepahydrate class of monoclinic crystal system in the space group $P 2_{1 / n}$ having unit cell dimensions $\mathrm{a}=9.2432(4) \mathrm{A}^{\circ}, \mathrm{b}=$ 10.9550(5) $\mathrm{A}^{\circ}$ and $\mathrm{c}=10.4216(5) \mathrm{A}^{\circ}$ and $\square=95.651(1) \mathrm{A}^{\circ}$ hydrated crystal of this kind is expected to have hydrogen bonding, as a result, these crystals are expected to have good nonlinear optical properties. The NLO property of this material is confirmed by Nd:YAG laser[13]. DSHP crystals found a number of scientific applications and specific uses in the industry $[14,15]$. It is thermally stable up to $800^{\circ} \mathrm{C}$ but there is an internal weight loss due to water of crystallization at lower temperature. It is some times difficult, with the X-ray crystallographic studies to determine the molecular structure and the associated space group, in particular when the possible structures differ mainly in the hydrogen positions. The postulated disorder involves predominantly the protons that are distributed between two equally populated sites. It is found that there is a disorder in the phosphate group in sodium hydrogen phosphate [16].

Potassium dihydrogen orthophosphate (KDP), $\mathrm{KH}_{2} \mathrm{PO}_{4}$ continues to be an interesting material both academically and industrially. KDP is a representative of hydrogen bonded crystals which possesses very good electro-optic and nonlinear optical (NLO) properties in addition to interesting electrical properties. Due to its interesting electrical and optical properties, structural phase transitions, and ease of crystallization, KDP has been the subject of a wide variety of investigations. The demand for high quality large KDP single crystals increases due to their application as frequency conversion crystal in inertial confinement fusion [17]. KDP belongs to the scalenohedral (twelve faced) class of tetragonal crystal system and is isomorphous to KDP [18]

A research programme has been planned in our laboratory to carry out a series of investigations on these materials at lower temperatures. As a part of our research programme we have grown higher quality DSHP single crystals and Potassium Dihydrogen Phosphate (KDP) doped with five different concentrations by the slow evaporation method. The objective of the present work was to investigate the effects of KDP in the normalized growth yield and electrical studies. Herein we report the results obtained.

\section{Experimental}

Analytical reagent (AR) grade DSHP, KDP and double distilled water were used for the growth of single crystals from aqueous solutions by using the procedure reported by John $\mathrm{N}$. J. et al [17] . In the same way DSHP was added with KDP in five different molar concentrations namely 1:0.002, 1:0.004,1:0.006, 1:0.008 and 1:0.010. The same molar concentration and temperature was maintained while preparing the doped crystals. The crystals were harvested after 20-30 days and shown in figure 1 .

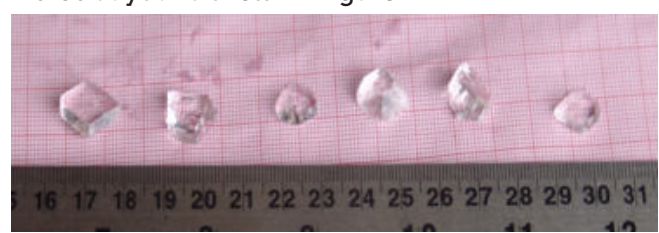

Figure 1: Photograph showing the pure and KDP added DSHP crystals grown

[From left are : 0.0 (pure DSHP), 0.2, 0.4, 0.6, 0.8 and 1.0 mole \% KDP added]

The melting point of the grown crystals was measured using a 
melting point apparatus ( model : tempo 120 ). When the crystals are grown in the same container in some medium, there is a possibility that the dielectric constant and related properties are different for different crystals. It would be better if there is a possibility to estimate quantitatively the concentration level of impurity that is present in each crystal. Hence, in order to understand qualitatively at least whether the added impurity has entered into the lattice or not, we carried out the density measurement by using the floatation technique [19-21]. Carbon tetrachloride of density 1.594 g./cc and bromoform of density $2.890 \mathrm{~g} / \mathrm{cc}$ were used for that purpose.

The sample crystals had a thickness about $4 \mathrm{~mm}$ and was polished for electrical measurements. Opposite faces of the sample crystals were coated with good quality graphite to obtain a good ohmic contact with the electrodes. The capacitance and dielectric loss $(\tan \square)$ were measured using the conventional two probe technique [22-24] at various temperatures ranging from 2 to $30 \circ \mathrm{C}$ using an LCR meter ( Model APLAB) with two frequencies, namely $100 \mathrm{~Hz}$ and $1 \mathrm{kHz}$. The dielectric constant was calculated using the relation

$\varepsilon_{\mathrm{r}}=\frac{\mathbf{C}_{\text {cry }}-\mathbf{C}_{\text {air }}\left(1-\left(\mathrm{A}_{\text {crys }} /{ }_{\text {Aair }}\right)\right)}{-C_{\text {air }}} \mathbf{x}\left(A_{\text {air }} / A_{\text {crys }}\right)$,

where Ccrys is the capacitance with crystal (including air), Cair is the capacitance of air, Acrys is the area of the crystal touching the electrode and Aair is the area of electrode. The AC electrical conductivity ( $\sigma \mathrm{ac}$ ) was calculated using the relation $\sigma a \mathrm{c}=\varepsilon 0 \mathrm{cr} \varepsilon \tan \varepsilon$ where $\varepsilon 0$ is the permittivity of free $\operatorname{space}(8.85 \times 10-12 \mathrm{Farad} / \mathrm{m})$ and $\varepsilon$ is the angular frequency $(\varepsilon$ $=2 \pi \mathrm{f}, \mathrm{f}=100 \mathrm{~Hz} \& \mathrm{lkHz})$.

The resistance of the crystals were measured using a thousand meg ohmmeter. The observations were made while heating the sample. The dimensions of the crystals were measured using a traveling microscope. The DC conductivity ( $\sigma \mathrm{dc}$ ) of the crystal was calculated using the relation $=\mathrm{d} /$ (RA) where $d$ is the thickness of the sample, $A$ is the area of the sample and $R$ is the measured resistance.

\section{Results and discussion}

All the crystals grown are found to be stable at room temperature, colourless, transparent and have well defined appearance. Since the temperature has not been completely kept constant during the growth of the crystals in the present work, there are morphological changes in the grown crystals. The melting point of pure DSHP crystal was found to be $35^{\circ} \mathrm{C}$. This is in good agreement with the reported value[17]. The impurity added DSHP also melts at this temperature. Therefore, KDP whose melting point is higher than DSHP [25] doesn't alter the melting point of the grown DSHP crystals. Figure 2 shows the variation in density values obtained in the present study. For the impurity considered in the present study the observed increase of density of DSHP crystal caused by the impurities indicates that the impurity molecules have entered into the lattice of DSHP crystals. Moreover it can be seen that the density increases further with the increase in impurity concentration of the aqueous solution of DSHP used for the growth of crystals. The observed density value of DSHP compares well with that reported in the literature[17].

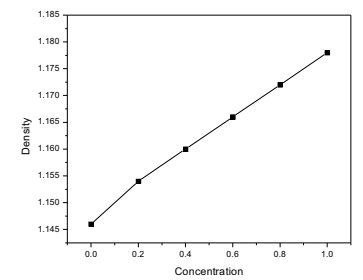

Fig. 2 :Variation of density with impurity
The term dielectric applies to the material properties governing the interaction between matter and electromagnetic field. Induced or permanent electric polarization or magnetization of matter as a function of static or alternating electric, magnetic or electromagnetic field constitutes the dielectric properties of the material. The dielectric constant is one of the basic electrical properties of solids. Various polarization mechanisms in solids such as atomic polarization of the lattice, orientational polarization of diploes and space charge polarizations can be understood very easily by studying the dielectric properties as a function of frequency and temperature for crystalline solids [26-28]. These investigations help in detecting the structural phase transitions taking place in solids when abrupt changes in dielectric properties are observed. Particularly the presence of a dielectric between the plates of a condenser enhances the capacitance. The effect makes material with dielectric constant useful in capacitor technology.

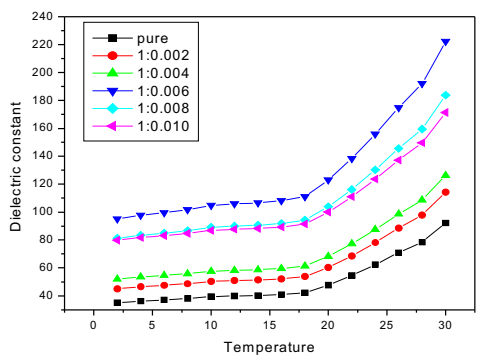

Fig. 3 :Variation of dielectric constant with temperature at $100 \mathrm{~Hz}$

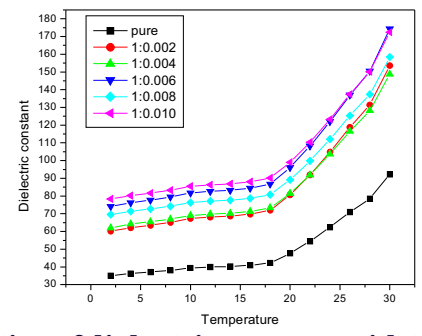

Fig. 4:Variation of dielectric constant with temperature at $1 \mathrm{kHz}$

The 8 values obtained for the grown crystals for $100 \mathrm{~Hz}$ frequency is provided in figure 3 and for $1 \mathrm{KHz}$ frequency in figure 4.The dielectric constant increases with the increase in temperature for both the frequencies. Variation of $\mathrm{\varepsilon r}$ with temperature is generally attributed to the crystal expansion, the electronic and ionic polarizations and the presence of impurities and crystal defects. The variation of $\mathrm{er}$ at low temperature is mainly due to the expansion and electronic and ionic polarization. At higher temperatures, the increase is mainly attributed to the thermally generated charge carriers and impurity dipoles.

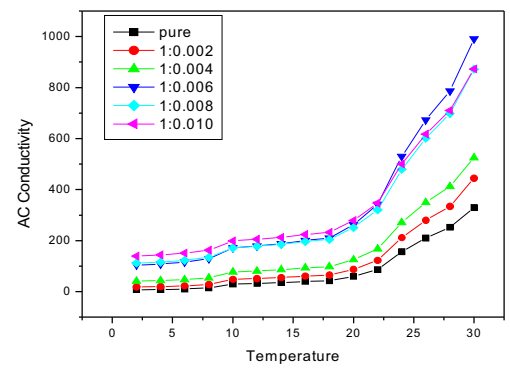

Fig. 5.Variation of dielectric loss with temperature at 100 $\mathrm{Hz}$

The dielectric loss tangent $(\tan \delta \quad$ ) is the imaginary part of dielectric constant and determines the loosiness of the medium. Similar to dielectric constant low loose tangent results in fast subtsract while large loss tangent results in a 
slow substract. The dielectric loss values obtained for the frequency $100 \mathrm{~Hz}$ is presented in figure 5 and for $1 \mathrm{~K} \mathrm{~Hz}$ in figure 6 . It increases with the increase of temperature. This may be attributed to the movement of halide ions in random directions.

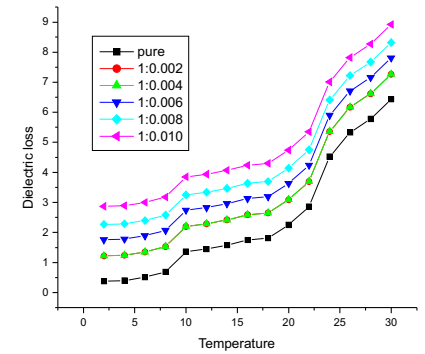

Fig. 6. Variation of dielectric loss with temperature at 1 $\mathbf{k H z}$

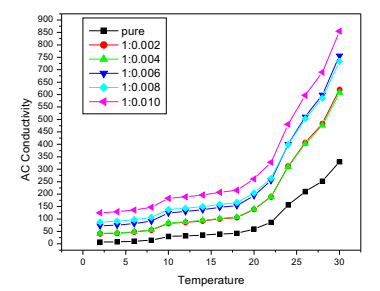

Fig.7: Variation of $\mathrm{AC}$ conductivity with temperature at $100 \mathrm{~Hz}$

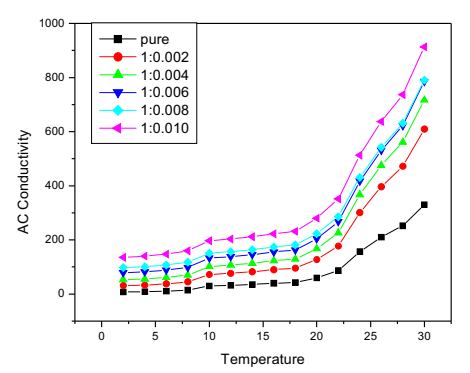

Fig. 8 Variation of $\mathrm{AC}$ conductivity with temperature at 1 kHz

The AC electrical conductivity ( $\sigma a c$ ) values obtained for all the six crystals for the two frequencies are provided in figure 7\&8. The AC electrical conductivity increases with increase in temperature and show no systematic variation with impurity concentration as in the case of dielectric constant and dielectric loss values. The reason may be due to the movement of halide ions in random directions, because of the ion-ion correlation which brings about the disorder. The values obtained for $\sigma$ dc are presented in Figure 9. The DC conductivity also increases with the increase in temperature.

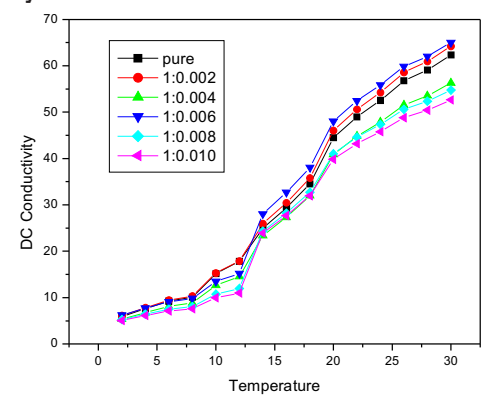

Fig. 9: Variation of DC electrical conductivities with temperature

\section{Conclusions}

Single crystals of pure and KDP added disodium hydrogen orthophosphate (DSHP) were grown by the slow evaporation method. The grown crystals are transparent and with well defined external appearance. Density measurements indicate that the KDP molecules have entered into the lattice of DSHP crystals. Melting point measurement indicates that the impurity doesn't alter the melting point. Capacitance and dielectric loss tangent $(\tan \delta)$ measurements were carried out for the grown crystals at various temperatures ranging from 2 to $30 \circ \mathrm{C}$ using an LCR meter with frequencies of $100 \mathrm{~Hz}$ and 1 $\mathrm{kHz}$. Dielectric constant and AC electrical conductivity were determined from the measured capacitance and $\tan \delta$ values. DC electrical conductivity was also measured at various temperatures. The dielectric constant, dielectric loss, AC conductivity, and DC conductivity are found to increase with increasing temperature for both the frequencies.

\section{References}

1. Joseph John N., Selvarajan P., Mahadevan C. K., "Growth and characterization of disodium hydrogen phosphate (DSHP) single crystals", Materials and Manufacturing Processes (2007) 22, 379-383. DOI: 10.1080/104269107 01190907

2. Joseph John N., Selvarajan P., Mahadevan C. K. "Studies on $\mathrm{NaCl}$ added ADP single crystals " Materials and Manufacturing Processes (2008) 23, 809-815. DOI: $10.1080 / 10426910802384573$

3. Joseph John N., Selvarajan P., Mahadevan C. K. "Growth, Structural, Optical, Mechanical and Dielectric Characterization of Diammonium Hydrogen Phosphate (DAHP) single Crystals", Journal of Minerals and Material characterization and Engineering, 10, 15,2011, 1379-1389.

4. Jayaprakash Manoharan A. J., Joseph John N., Andavan P. " Effect of amino acid doping on the dielectric properties of triglycine sulphate (TGS) single crystals", Ind.J. of Sci. and Tech., 4,6,2011,688-691.

5. Jayaprakash Manoharan A. J., Joseph John N., Andavan P. "Dielectric properties of proline doped Triglycine sulphate (TGSP) crystals, Journal of Experimental Sciences, (2011),2(2),33-35.

6. Sivakala P. and Joseph John N. and S. Perumal "Investigation on the Growth and Physio-Chemical Properties of L-Alanine Mixed BTCBC Single Crystals" Int. Journal of Engineering Research and Applications, Vol. 4, Issue 7(Version 4), 2014,pp.145-151.

7. Sivakala P. and Joseph John N. and S. Perumal "Growth and Physio-Chemical Properties of pure and L-Alanine admixed TTZP Single Crystals" Int. res. J. Eng.And Tech., 3,2,2016, 1273-1278.

8. on Ferroelectric Material L-proline doped TGS single crystals for IR detectors",Int.J.Current Sci.8,11,2016,41068-41074

9. Joseph John N. "Dielectric properties of ferroelectric L-proline triglycine sulphate (LPTGS) crystal"--Int.J.Innov. Sci. and Res. 5, 11, 2016, 890-893.

10. Praveen P G, Ayarine G Dhas, Joseph John N., Investigation on the Effect of lithium Lactate on the Electrical Properties of TGS Single Crystals", Stard Research, 7,10,2020,1017-1024.https://doi.org/10.37896/sr7.10/108

11. M. Shanmugam, F. D. Gnanam, P. Ramasamy, Indian J. Pure Appl. Phys. 23 (1985) 82.

12. Mugundakumari S., Priya K., Thangaraj N., Rakesh R., Joseph John N., "Growth and characterization of $\mathrm{CaCl} 2$ doped organometallic L-aspargine Cadmium Chloride Monohydrate (LACCM) crystals, Int.J. Current Research, 2017, 9,6, 51830-51834.

13. Gunasekaran M.;Vijayan N.; Ramesh Babu R.; Gopalakrishnan R.; Pamasamy P.; Lan C.W.,J. "Growth and characterization of DSHP crystals", Crys. Growth. 244 (2002) 194-199

14. Mugundakumari S., Thangaraj N., Rakesh R., Joseph John N., "Growth and characterization of $\mathrm{NaCl}$ doped organometallic L-aspargine Cadmium Chloride Monohydrate (LACCM) crystals, Int. J. Current Research and Review,2017,9,12,11-17, doi.org/10.7324/IJCRR.2017.9122

15. Baldus, M.; Meier, B.H.; Ernst, R.R.; Kentgens, A.P.M.; Meyer Zu Altuschildesche,H.;Nesper, R., J.Am. Chem. Soc., 117(1995)5141-5147.

16. Suresh D. M., Joseph John N, "'Growth, Thermal and Mechanical Properties of Disodium Hydrogen Phosphate (DSHP) single Crystals", Int. Res.J. Eng. Tech., 5,1,2018.313-316

17. Wyckoff,R.W.G. Crystal Structures (Vol.3) (second edn.);Interscience :New York, 1960

18. Suresh D. M., Joseph John N, "Electrical conductivity measurements on pure and Magnesium chloride added Disodium Hydrogen Phosphate (DSHP) single Crystals", Scholars Journal of Physics, Chemistry and Statistics, 5(1), 2018,78-84.

19. Ayarine G.Dhas, Ramalingam A., Suresh D. M., Asbalter J., and Joseph John N. "Electrical Behaviour of Aminoacid doped TGS single crystals", Journal of Applied Science and Computations, 5, 7,2018,469-475. ISSN : 1076 - 5131

20. Mugundakumari S., Thangaraj N., Rakesh R., Praveen P. G., Joseph John N., "Growth and characterization of organometallic L-aspargine Cadmium Chloride Monohydrate (LACCM) crystals, Journal of emerging technologies and innovative research,2018,5,8,159-165

21. Ayarine G.Dhas, Rakesh R., Thangaraj N., Asbalter J., Joseph John N., "Studies on Glutamic acid doped TGS single crystals" , Journal of Engineering Research and Applications, 8,8,2018,45-50.Doi:10.9790/9622-0808034550

22. Praveen P G., Ayarine G. Dhas, Rakesh R. , Thangaraj N. , Joseph John N., "Electrical conductivity measurements on undoped and ADP doped Disodium Hydrogen Phosphate (DSHP) single crystals" , International Journal Engineering Science Innovation, 7,11(1),2018, 18-24.

23. Praveen P G., Ayarine G. Dhas, Rakesh R. , Thangaraj N. , Joseph John N., "Dielectric measurements on undoped and urea added DSHP single crystals", IOSR Journal of Applied Physics", 10,6(1), 2018, 21-25. DOI: $10.9790 / 4861-1006012125$

24. John A. Dean.,(1973) Lange's Hand book of Chemistry; (Eleventh Editon)[ McGraw-Hill Book Company:NewYork]

25. Ayarine G. Dhas, Joseph John N., "Effect of l-aspargine on Structural, Photoluminescence and Photoconductive propereties of Triglycine Sulphate single crystals", The International Journal of Analytical and Experimental Model Analsis, 1 1,9,2019, 1250-1267. ISSN : 0886-9367. 
26. Govinda S., Rao K.V., "Dielectric Properties of Single Crystals of Al2O3 and Al2O3 Doped with Chromium or Vanadium" (1975) Phys. Stat. Solidi. 27, 639. doi.org/10.1002/pssa.2210270237

27. Ayarine G.Dhas, Joseph John N.," Investigation on the effect of 1-cysteine on the ferroelectric properties of TGS crystal ", Journal of Information and computational Science, 9,11, 2019. Doi:10.12733.JICS.2019.V9I11. 535569.1131

28. Meena, M.; Mahadevan, C.K. "Growth and Characterization of L-arginaine added KDP and ADP single crystals", Cryst. Res.Technol.2008,43, 166-1 72 . doi.org/10.1002/crat.200711064

29. Praveen P G, Ayarine G Dhas, Joseph John N., Growth and Characterization of Sodium Lactate added TGS Single Crystals", Compliance Engineering Journel, 11,20,2021,390-399.

30. Wyckoff,R.W.G. Crystal Structures (Vol.3) (second edn.);Interscience :New York, 1960 